\title{
Case Report: Can Steroids Help in a Case of Allodynia Following Spinal Anaesthesia for a Caesarean section?
}

\author{
Reham Mahrous $^{1 *}$, Doaa Alaa El dien Abd El Fattah', Ahmed Hasanin ${ }^{3}$, Mohamed Ahmed Ibrahim ${ }^{4}$, \\ Amr K Abdelhakeem ${ }^{5}$ \\ 1,4,5 Lecturer of Anaesthesia, Surgical ICU, ${ }^{2}$ Lecturer of Obstetrics and Gynaecology Department, \\ ${ }^{3}$ Assistant professor of Anaesthesia, Surgical ICU, ${ }^{1,2,3,4,5}$ Cairo University
}

\begin{abstract}
Most of the anaesthesia related neurological complications are associated with paresthesia upon introduction of the spinal needle. Steroids may shorten the duration of the effects caused by nerve injury. A 27-year-old female patient undergoing caesarean section experienced paresthesia during the intrathecal introduction of the spinal needle. After the spinal anaesthesia wore off, she developed tactile allodynia. Intravenous hydrocortisone was given; marked improvement of pain and allodynia was observed. Contact of the needle with a nerve root may have caused an inflammatory response. The benefit of steroids in cases of nerve injury is still debatable. They likely decrease the levels of autoantibodies that delay nerve regeneration.
\end{abstract}

Keywords: allodynia, caesarean section, hydrocortisone, paresthesia, spinal anaesthesia, steroids

\section{Introduction}

Spinal anaesthesia is commonly used in caesarean section operations with relatively less complications as compared to general anaesthesia. Neurological complications of spinal anaesthesia are not common. ${ }^{1}$ Allodynia is defined as painful sensation that results from a non-painful stimulus. It usually happens in conditions associated with nerve damage such as diabetes mellitus. ${ }^{2}$ Apart from a few case reports, there is limited information in the literature regarding allodynia as it relates to spinal anaesthesia.

*Correspondence: Reham Mahrous

E mail: dr_memoo2003@hotmail.com

Received: 29/10/2020

Accepted: 21/08/2021

DOI: https://doi.org/10.4038/slja.v29i2.8703

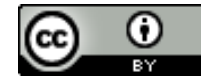

Most neurological complications during spinal anaesthesia are associated with paresthesia upon introduction of the spinal needle or pain with injection of the local anaesthetic. ${ }^{3}$ One large prospective survey conducted in 1997 found that most of the patients who had neurologic deficits experienced paresthesia during the insertion of the spinal needle or pain with injection. ${ }^{4}$

Steroids may play a role in shortening the duration of the effects caused by nerve injury, by reduction of neural edema or promoting recovery of nerve functions. ${ }^{5}$

Here, we present you with a case of a 27-year-old primigravida patient with post anaesthesia allodynia that was successfully treated with IV hydrocortisone.

\section{Case report}

A 27 years old primigravida, weighing $55 \mathrm{~kg}$ and a height of $164 \mathrm{~cm}$ was scheduled for an elective caesarean section, with no history of medical diseases or previous operations. The patient 
consented to spinal anaesthesia based on her personal preference.

After application of standard monitoring and securing intravenous access, intrathecal anaesthesia was given in the sitting position. A 25 gauge beveled cutting needle was used at the level of lumbar $4 / 5$ intervertebral space. The patient experienced paresthesia during introduction of the needle. Further introduction of the needle was stopped, and trocar was removed. When the patient confirmed that the paresthesia had completely disappeared, the cerebrospinal fluid was checked for free flow and free barbotage and, fentanyl $25 \mathrm{mcg}$ and hyperbaric bupivacaine $(0.5 \%)$ 10mg were injected.

After confirming adequate level of blockade, the operation was lasted for an hour. No significant events or hemodynamic changes were observed intraoperatively. After the surgery, the patient was discharged to the post-anesthesia care unit and then to her room. Morphine 5mg 8 hourly was prescribed for post-operative analgesia. Two hours later, the patient complained of severe pain on the medial aspect of the right foot with marked tactile allodynia. The patient was given a single dose of hydrocortisone 100mg intravenously and followed up for the next 48 hours.

Forty-five minutes later, the pain dramatically improved with disappearance of allodynia. After two hours, the patient was able to walk with minimal pain, and within six hours the pain completely disappeared. During follow up visits in the two weeks period after discharge and continuous telecommunication follow up, the patient confirmed that the pain never re-appeared.

\section{Discussion}

Allodynia is defined as the experience of pain from a non-painful stimulus. Our patient experienced paresthesia during the introduction of the spinal needle and developed tactile allodynia as the spinal anaesthesia was wearing off. Although Paresthesia is more common during introduction with pencil point spinal needles, the incidence with cut needle is still considerable. ${ }^{3}$ This may be the result of the spinal needle coming in contact with a nerve root in the subarachnoid space, epidural space, or within the intervertebral foramen, ${ }^{6}$ which may cause an inflammatory response. Therefore, we considered using corticosteroids to reduce paresthesia, the patient was experiencing.

Our patient experienced allodynia with the use of intrathecal bupivacaine, which is a rare occurrence based on a study by John M. Freedman et al. which compared the incidence of paresthesia with different local anesthetics in 1863 patients. $^{7}$

The use of steroids in cases of nerve injury is debatable, as they are believed to decrease the levels of autoantibodies, which may have a deleterious effect on nerve regeneration. ${ }^{8}$ However, Lieberman DM et al. found that, following facial nerve injury, adult mice that were treated with corticosteroids recovered much slower than those who received normal saline injections. $^{9}$

While allodynia is relatively rare, there are instances where corticosteroids have improved nerve function after potential direct mechanical insult. In a study that examined the recovery of recurrent laryngeal nerve injury after thyroidectomy, it was found that the prophylactic use of corticosteroids shortened the recovery time. $^{5}$

A case report of a patient who had foot drop after spinal anaesthesia for a caesarean section operation showed that, the use of corticosteroids and anti-inflammatory drugs showed complete recovery within a few days, and no observed side effects. ${ }^{10}$ Based on these findings, we decided on intravenous hydrocortisone treatment for our patient.

This case report highlights the potential of corticosteroid therapy in those patients who present with post anesthesia related allodynia. Intravenous hydrocortisone provided effective relief of pain without any side effects. 


\section{References}

1. Freshour CR, Constance D. ASRA Practice Advisory on Neurologic Complications in Regional Anesthesia and Pain Medicine. NIH Public Access. 2018; 33(5):404-15.

2. Lolignier S, Eijkelkamp $\mathrm{N}$, Wood JN. Mechanical allodynia [Internet]. Vol. 467, Pflugers Archiv European Journal of Physiology. Springer Verlag; 2014 [cited 2020 Oct 28]. p. 133-9. Available from: https://pubmed.ncbi.nlm.nih.gov/24846747/

3. Imbelloni LE, Pitombo PF, Ganem EM. The Incidence of Paresthesia and Neurologic Complications after Lower Spinal Thoracic Puncture with Cut Needle Compared to Pencil Point Needle. Study in 300 Patients. 2010; 1(2):2-5.

4. American Society of Anesthesiologists. Y, Narchi P, Messiah A, Litt L, Rouvier B, Samii K. Anesthesiology. [Internet]. Vol. 87, Serious complications related to regional anesthesia: results of a prospective survey in France. [American Society of Anesthesiologists, etc.]; 1997 [cited 2019 Jun 5]. 479-486 p. Available from: http://anesthesiology.pubs.asahq.org/article.a spx? articleid $=1948141$

5. Wang LF, Lee KW, Kuo WR, Wu CW, Lu SP, Chiang FY. The efficacy of intraoperative corticosteroids in recurrent laryngeal nerve palsy after thyroid surgery. World J Surg. 2006; 30(3):299-303.

6. Gmelch BS, Bernards CM. Does a Paresthesia During Spinal Needle Insertion Indicate Intrathecal Needle Placement? 2009; 34(1):29-32.

7. Freedman JM, Li DK, Drasner K, Jaskela MC, Larsen B, Wi S. Transient neurologic symptoms after spinal anesthesia. Am epidemiologic study of 1,863 patients. Anesthesiology. 1998 Sep 1; 89(3):633-41.

8. Lee SK, Wolfe SW. Peripheral Nerve Injury and Repair. 2000; 8(4):243-52.

9. Lieberman DM, Jan TA, Ahmad SO, Most SP. Effects of Corticosteroids on Functional Recovery and Neuron Survival After Facial Nerve Injury in Mice. 2011; 13(2):117-24.

10. Razavi M. oot drop after spinal anesthesia for cesarean section : a case report. 2018; 45-7. 